\title{
CRYSTAL: a multi-agent Al system for automated mapping of materials' crystal structures
}

\author{
Carla P. Gomes, Junwen Bai, Yexiang Xue ${ }^{a)}$, Johan Björck, Brendan Rappazzo, Sebastian Ament, Richard Bernstein, and \\ Shufeng Kong, Department of Computer Science, Cornell University, Ithaca, NY 14853, USA \\ Santosh K. Suramb), Joint Center for Artificial Photosynthesis, California Institute of Technology, Pasadena CA 91125, USA \\ R. Bruce van Dover, Department of Materials Science and Engineering, Cornell University, Ithaca, NY, USA \\ John M. Gregoire (D, Joint Center for Artificial Photosynthesis, California Institute of Technology, Pasadena CA 91125, USA
}

Address all correspondence to Carla P. Gomes at gomes@cs.cornell.edu and John M. Gregoire at gregoire@caltech.edu

(Received 18 January 2019; accepted 8 April 2019)

\begin{abstract}
We introduce CRYSTAL, a multi-agent Al system for crystal-structure phase mapping. CRYSTAL is the first system that can automatically generate a portfolio of physically meaningful phase diagrams for expert-user exploration and selection. CRYSTAL outperforms previous methods to solve the example Pd-Rh-Ta phase diagram, enabling the discovery of a mixed-intermetallic methanol oxidation electrocatalyst. The integration of multiple data-knowledge sources and learning and reasoning algorithms, combined with the exploitation of problem decompositions, relaxations, and parallelism, empowers Al to supersede human scientific data interpretation capabilities and enable otherwise inaccessible scientific discovery in materials science and beyond.
\end{abstract}

\section{Introduction}

Artificial Intelligence (AI) excels at a range of cognitive tasks, from speech and image recognition to game playing, ${ }^{[1,2]}$ and holds great promise for automating scientific discovery. ${ }^{[3-8]}$ The interpretation of scientific data remains a challenge for AI due to both the need for intricate scientific background knowledge and reasoning and the lack of large annotated training datasets. AI-based reasoning and learning methods are particularly critical for the field of high-throughput materials science where automated experiments are dramatically accelerating the pace of materials discovery for a variety of critical technologies. ${ }^{[4-6,9,10]}$ Foundational techniques in highthroughput materials discovery include simultaneous synthesis of hundreds to thousands of materials using co-sputtering followed by rapid structural characterization via synchrotron XRD [Fig. 1(a)]. For complex materials containing three or more elements, the most common rate-limiting step in the discovery process is the construction of a crystal phase diagram from the composition and structural characterization data. We refer to this task as the phase mapping problem, which requires the identification of basis patterns (or factors) corresponding to pure crystal phases, some of which may not be sampled separately, such that all the XRD measurements can be explained

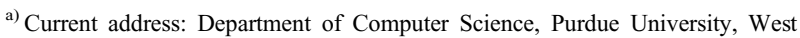
Lafayette, IN 47907, USA.

b) Current address: Toyota Research Institute, Los Altos, CA 94022, USA.
}

as a mixture of the basis patterns [Figs. 1(c)-1(f)]. The XRD measurements are typically noisy, which contributes to the challenge of separating the basis pattern "sources" from the collection of patterns. Additionally, materials thermodynamics places a set of intricate physical constraints on the solution, and while synthesis of materials may not reach thermodynamic equilibrium, the non-equilibrium behavior is most commonly exhibited as the presence of non-equilibrium phases as opposed to deviations from, e.g., the Gibbs phase rule.

Phase mapping has traditionally been a bottleneck of the high-throughput materials discovery cycle as the synthesis and characterization experiments [Figs. 1(a) and 1(b)] can be performed on several libraries of materials per day while the manual effort required to solve a given phase mapping problem limits the throughput to only several phase diagrams per year. Previous reports have detailed the shortcomings of existing de-mixing algorithms, ${ }^{[12]}$ most notably in the presence of noise and substantial alloying, an important phenomenon in which a range of elemental compositions crystallize into the same phase, causing its basis pattern to shift systematically with composition. ${ }^{[13]}$ Non-negative matrix factorization (NMF) techniques ${ }^{[14]}$ have shown promise in the efficient extraction of representative diffraction patterns from large datasets, ${ }^{[15,16]}$ but their limited ability to encode physical constraints and prior knowledge results in routine production of non-physical solutions. From a computational perspective, phase mapping is an example of a challenging NP-hard prob$\mathrm{lem}^{[17]}$ whose sheer number of possible combinations of 
(a) Library synthesis/characterization Combinatorial sputter deposition

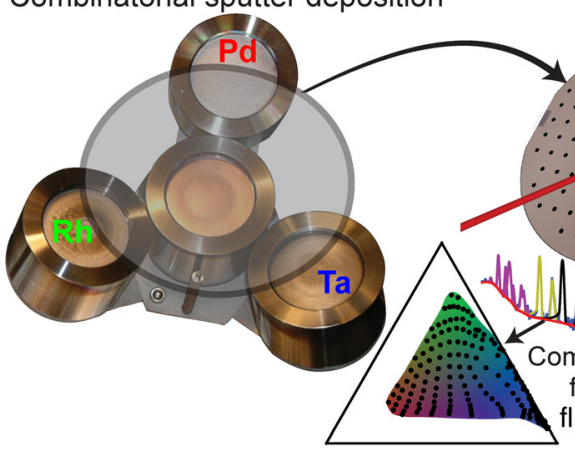

(c) Phase mapping problem

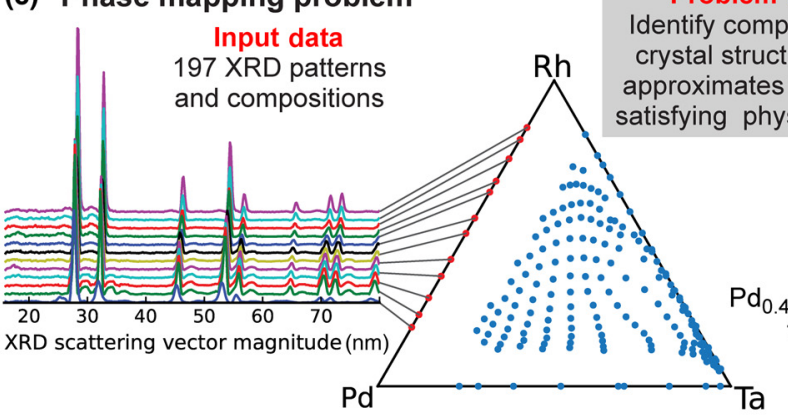

(b) Parallel catalyst screening

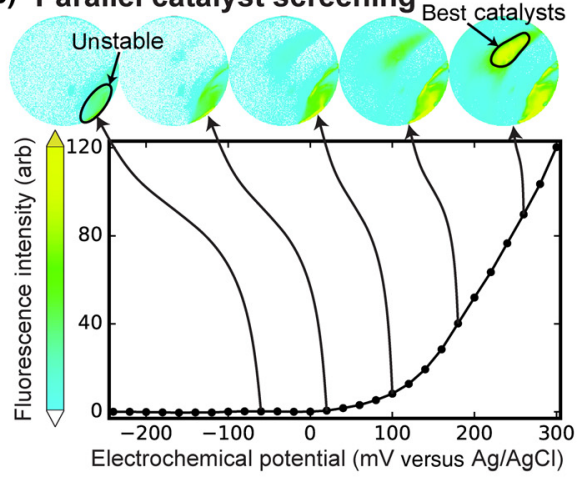

CRYSTAL's analysis and data Interpretation Phase fields: sets of coexisting phases (e) fcc structure match

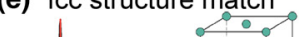

(d) sics constraints (d)

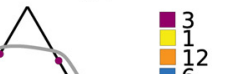

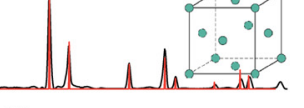

(f) fcc pattern shifting
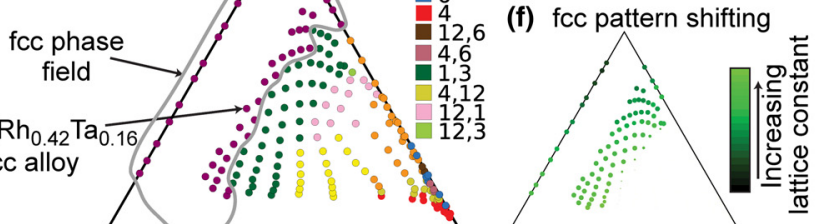

Figure 1. Materials discovery cycle. (a) Synthesis of materials using sputter co-deposition from palladium (Pd), rhodium (Rh), and tantalum (Ta) sources to form a "materials library" thin film with continuous composition variation. Collection of both elastically (XRD) and inelastically (XRF) scattered x-rays, using a synchrotron $\mathrm{X}$-ray beam, to characterize the materials' crystal structure and composition, respectively, the latter enabling a ternary composition map of the Pd-Rh-Ta library. (b) Each library is screened for catalytic activity using an electrochemical imaging strategy in which the best catalysts are identified using a fluorescent marker. ${ }^{[11]}$ Materials which appear active in the absence of methanol are denoted as "unstable." (c) The triangle-composition plot contains the 197 distilled XRD/XRF measurements that comprise the input for phase mapping. The 12 XRD patterns along the Pd-Rh composition line illustrate

composition-dependent peak shifting due to the two elements alloying in a single-crystal structure. (d) CRYSTAL's phase map solution identifies five phases (purple, yellow, orange, blue, and red) and six multi-phase fields; each sample's XRD pattern is explained by either a single phase or a mixture of phases. (e) The XRD pattern for a phase "3" sample is shown with red sticks denoting the known peak pattern of the face centered cubic (fcc) crystal structure, indicating that the broad range of compositions in the fcc phase field crystallize into this same structure. (f) The average atomic radius varies systematically with the alloy composition, which CRYSTAL captures by mapping the composition-dependent fcc lattice constant.

basis patterns and activations grows exponentially with data size, rendering monolithic solvers and traditional search methods computationally infeasible, which motivates the exploration of innovative AI approaches.

\section{CRYSTAL: a multi-agent AI system for phase mapping}

CRYSTAL is a multi-agent AI system that can run in unsupervised or semi-supervised mode and that decomposes phase mapping into smaller, more tractable sub-problems that are tackled by nimble algorithmic bots with unique background knowledge and reasoning capabilities (Fig. 2). Interleaved Agile Factor Decomposition (IAFD) is CRYSTAL's core phase-mapping engine, which interleaves factor decomposition (AgileFD bot) with constraint enforcement (Gibbs, Gibbs Alloy, and Phase Connectivity bots), whose collective reasoning produces physically meaningful phase maps. At a high level, IAFD relaxes and postpones the combinatorial physical constraints and iteratively repairs and enforces them when violations are detected.

The graph reasoning algorithms of the Gibbs, Gibbs Alloy, and Phase Connectivity bots are applied at a local scale to enable parallel computation and ensure scalability for large real-world problems. The key insight is that global maintenance of the combinatorial physical constraints is computationally prohibitive, yet appropriate data exploitation with local constraint enforcement provides global constraint satisfaction at a relatively small computational expense. While generating a phase diagram is a confounding and time-consuming task even for experienced materials scientists, IAFD generates a solution typically within 2 min for the dataset reported in this paper, a groundbreaking advance in phase mapping since no other algorithm imposes the physical constraints to reliably yield physically meaningful solutions (see Table S1).

The capability to rapidly generate physically meaningful solutions enables CRYSTAL's large-scale computations to assess solution stability, uncovering a critical and previously 


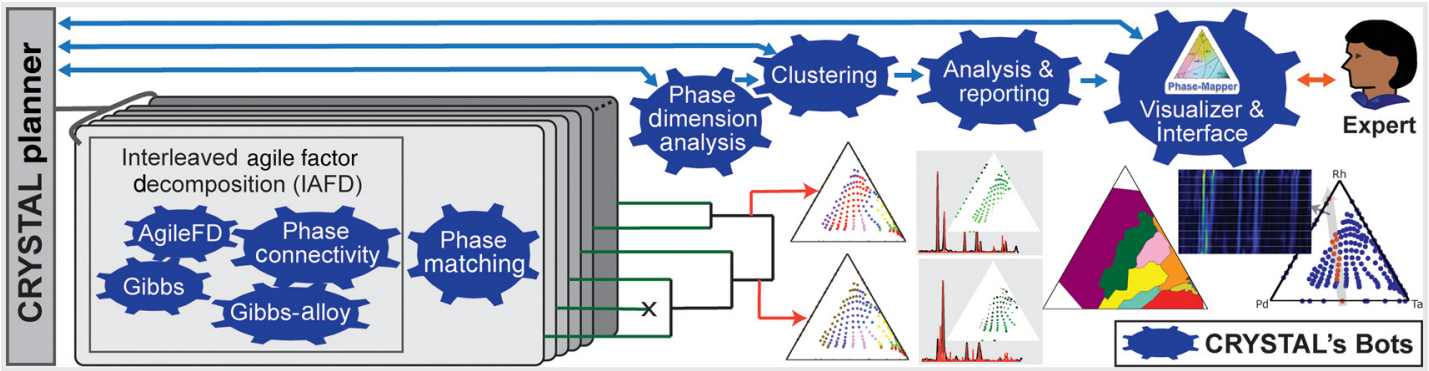

Figure 2. Outline of the CRYSTAL system. CRYSTAL incorporates a diverse collection of fast and specialized algorithms with different types of knowledge and computational capabilities. IAFD integrates the AgileFD, Gibbs, Gibbs Alloy, and Phase Connectivity bots, constituting CRYSTAL's core phase-mapping engine: AgileFD performs agile factor decomposition to learn the factors or basis patterns, corresponding to pure crystal structures, and its three partner bots enforce physical constraints. The Phase Matching bot matches the basis patterns discovered by IAFD to known crystal structure patterns from databases. The Phase Dimension Analysis bot analyzes and validates the generated phase maps and infers the system's maximum number of pure phases, which dictates how many system configurations CRYSTAL explores, using parallelism and randomization, to produce a large number of candidate phase maps. The hierarchical Clustering bot uses automated thresholding to identify a small set of representative candidate solutions, which are provided to either the CRYSTAL Planner for solution refinement or to the Analysis \& Reporting bot to generate phase diagrams and other visualizations for human-expert inspection. The Visualizer \& Interface bot enables users to interact with CRYSTAL for solution selection and fine tuning.

overlooked aspect of phase mapping. Even with the imposed physical constraints, different phase diagram solutions are often "inadequately differentiated" by the source XRD patterns. Inadequate differentiation in phase mapping arises in part from the fundamental non-invertibility of an XRD pattern to obtain its crystalline phase source(s) and is compounded by both noise in the source data and presence of different phases with similar basis patterns. The standard practice in phase mapping, and more generally in data modeling, is to extract knowledge from a single solution that sufficiently reconstructs the source data. In contrast, CRYSTAL explores the search space by deploying bots in parallel to produce a large number of candidate solutions. Using additional bots for solution analysis and aggregation (Figs. 2-4), CRYSTAL runs unsupervised and autonomously to generate a parsimonious portfolio of phase diagrams that represent different interpretations of the source data.

\section{CRYSTAL's algorithms}

As mentioned above, CRYSTAL is a collection of nimble algorithmic bots, with different knowledge and reasoning capabilities performing a variety of tasks outlined in Fig. 2 and described in more detail below. The IAFD bots collectively solve the phase mapping problem, using an unsupervised generative approach, to produce a phase map that satisfies the physical constraints. The CRYSTAL planner launches parallel runs of IAFD with different random initializations and parameters, in particular the number of target phases, and each IAFD run follows the algorithm outlined below and illustrated in Fig. 3:

Step 0: Initialization: Initialize the inner-loop (AgileFD-Gibbs) counter CNT1, bounded above by $p$, together with CNT2 which counts outer-loops bounded above by $q$, to be 0 . As discussed below, $p$ and $q$ are typically set to 3 and 2 respectively.
Step 1: AgileFD bot: Apply AgileFD on randomly selected $N / p$ "untouched" samples

Step 2: Gibbs bot: Enforce Gibbs phase constraint on these $N / p$ samples

Step 3: If some samples still have not been processed, namely CNT1 is still smaller than $p$, increase CNT1 by 1 and go back to step 1, otherwise, move on to step 4

Step 4: Gibbs-Alloy bot: enforce Gibbs-Alloy constraint on all $N$ samples

Step 5: Phase Connectivity bot: enforce Connectivity constraint on all $N$ samples

Step 6: If Gibbs-Alloy constraint is violated, go back to step 4, otherwise, go to step 7

Step 7: If CNT2 hasn't reached the upper bound $q$, refined solutions from Step 6 are fed into step 1 as initialization and the whole algorithm starts over again. Otherwise, the IAFD output is taken as the phase map finalized in step 6. Further documentation and source code for IAFD can be found at http://www. udiscover.it/resources/software/.

\section{AgileFD bot}

As illustrated in Fig. 3, we formulate phase mapping as a constrained matrix factorization problem. Experimental XRD measurements are represented by a matrix $\mathbf{A}$ of size $\mathrm{L} \times \mathrm{N}$. Each column of $A$ is a vector representing the XRD pattern sampled at $L$ diffraction angles, obtained at one out of $N$ sample locations. The phase mapping problem entails decomposing $\mathbf{A}$ in terms of factors $\mathbf{W}$ and $\mathbf{H}$, satisfying physics constraints. $\mathbf{W}$ encodes the characteristic patterns or structure associated with each pure crystalline phase and $\mathbf{H}$ represents the mixing parameters, such as the phases present, their proportions, and any alloying present.

Under the assumed "isotropic" alloying model, an XRD pattern measured as a function of scattering vector magnitude will 


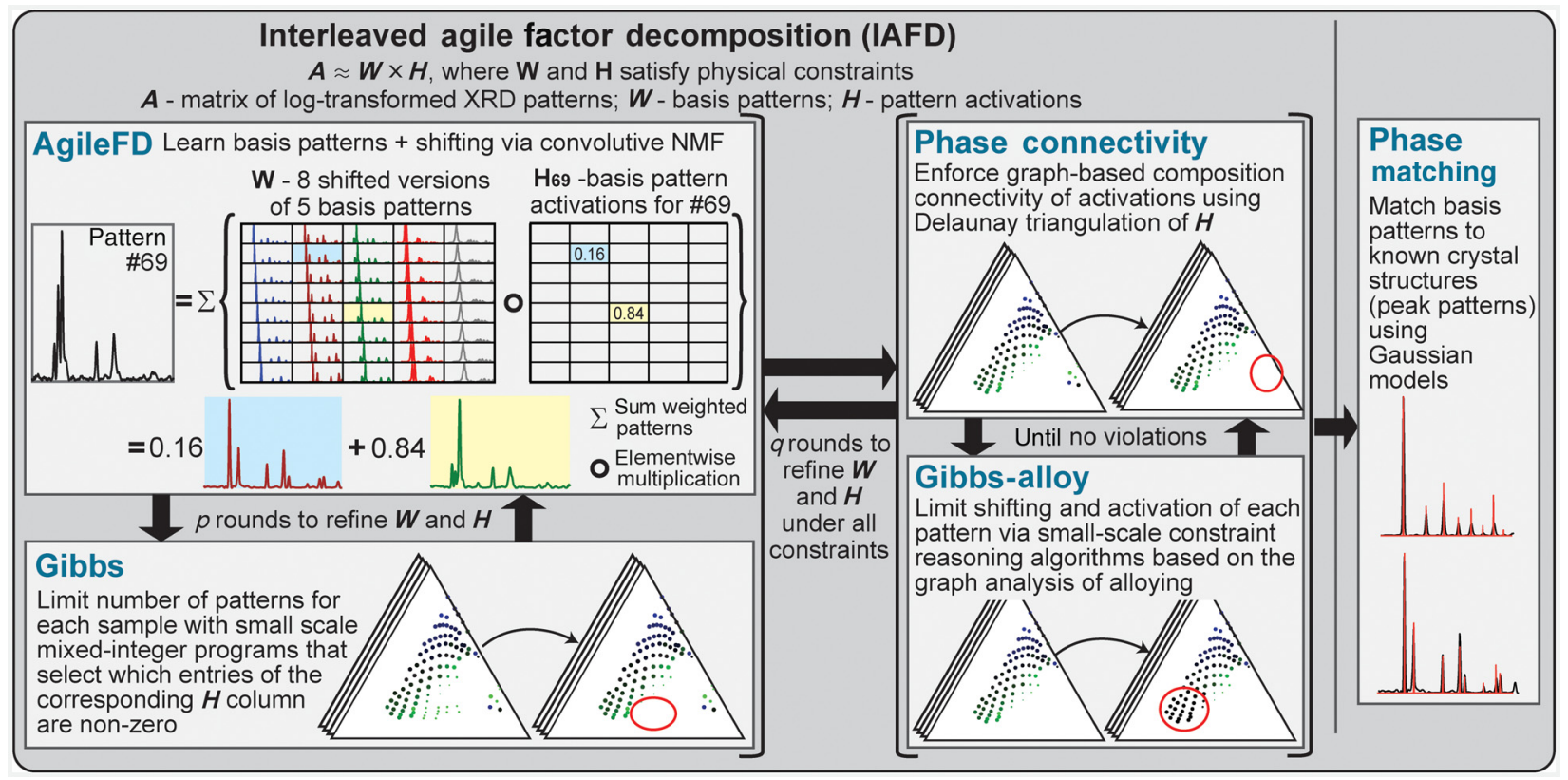

Figure 3. Solving phase mapping using an unsupervised generative approach. The IAFD bot network solves phase mapping as a constrained matrix factorization problem in which the input XRD pattern matrix $(\mathbf{A})$ is decomposed into factors $\mathbf{W}$ and $\mathbf{H}$ such that $\mathbf{W} \times \mathbf{H}$ approximates $\mathbf{A}$ while satisfying physical constraints. $\mathbf{W}$ encodes the characteristic patterns of pure crystal phases (including shifted versions) and $\mathbf{H}$ their activations, which dictate both the amount and the pattern shifting extent of each pure phase in each XRD measurement. IAFD starts with $p$ (typically three) rounds of interactions between the AgileFD and Gibbs bots followed by rounds of iterations between the Gibbs Alloy and Phase Connectivity bots, until all the constraints are satisfied. AgileFD performs matrix factorization using light-weight multiplicative updating rules, without enforcing the combinatorial physical constraints. The AgileFD solution's violations of the connectivity constraint and the constraints based on Gibbs' phase rule are repaired by the corresponding bots in an interleaved manner using efficient algorithms (red circles highlight repaired activations of $\mathbf{H}$ ). The entire procedure is repeated for solution refinement (typically $q=2$ ), and the resulting generated basis patterns are passed to the Phase Matching bot to identify the crystal structures by comparison with ICDD and/or determine if the solution potentially contains a new phase. The figure illustrates a representative XRD pattern of the Pd-Rh-Ta system (\#69) that is decomposed into shifted versions of two different basis patterns (0.16 and 0.84 of each, respectively).

shift multiplicatively, with, for example, a $1 \%$ lattice contraction causing peaks to shift by a factor of 1.01 . We convert the multiplicative shift to an additive constant by performing the factorization of logarithmically transformed patterns, making $\mathbf{A}$ a convolutive mixture of the bases in $\mathbf{W}$. $\mathbf{W}$ and $\mathbf{H}$ are naturally non-negative, leading to a convolutive non-negative matrix factorization (CNMF) problem, ${ }^{[18]}$ which AgileFD performs using lightweight multiplicative coordinate gradientdescent updating rules applied to the logarithmically transformed XRD data. ${ }^{[19,20]}$

\section{Gibbs and Gibbs-Alloy bots}

For a physical system where $l$ elements are deposited, Gibbs' phase rule implies that at most $l$ phases are present at each sample location. Mathematically, this is equivalent to constraining the number of non-zero elements in the vector $\sum_{m} \mathbf{H}_{*, n}^{m}$ for any sample location $n$ to be no more than $l$.

The Gibbs bot uses a Mixed Integer Programming (MIP) approach to find the best activation matrix $\mathbf{H}$ that activates no more than $l$ phases per sample point and minimize the reconstruction loss, while holding the phases in $\mathbf{W}$ fixed. ${ }^{[21]}$ Notice that when $\mathbf{W}$ is fixed, the columns of matrix $\mathbf{H}$ are independent which leads to a decomposition into a smaller MIP program per violated sample point, which finds the best $l$ phases that minimize the reconstruction loss of sample location $n$, as described further in the Supplementary Information.

The Gibbs Alloy bot extends the Gibbs constraint by reducing the allowed number of coexisting phases allowed by one when alloying is detected, i.e., for those sample locations with varying mean shift parameters compared with nearby locations. This is motivated by the thermodynamic degree of freedom associated with alloying, although instead of identifying details of the alloying behavior, the bot identifies where alloying is taking place and lowers the number of allowable phases by 1 at those composition points.

\section{Phase and Phase Field Connectivity bot}

The Connectivity constraint requires that both (i) the sample points where a specific phase is present and (ii) the sample points where each unique set of phases is present form a connected component in composition space, which is determined using the activations of each phase for each composition sample. Specifically, we define a graph $G$ in which sample points are nodes and two nearby sample points in composition space are connected with an edge based on the Delaunay 


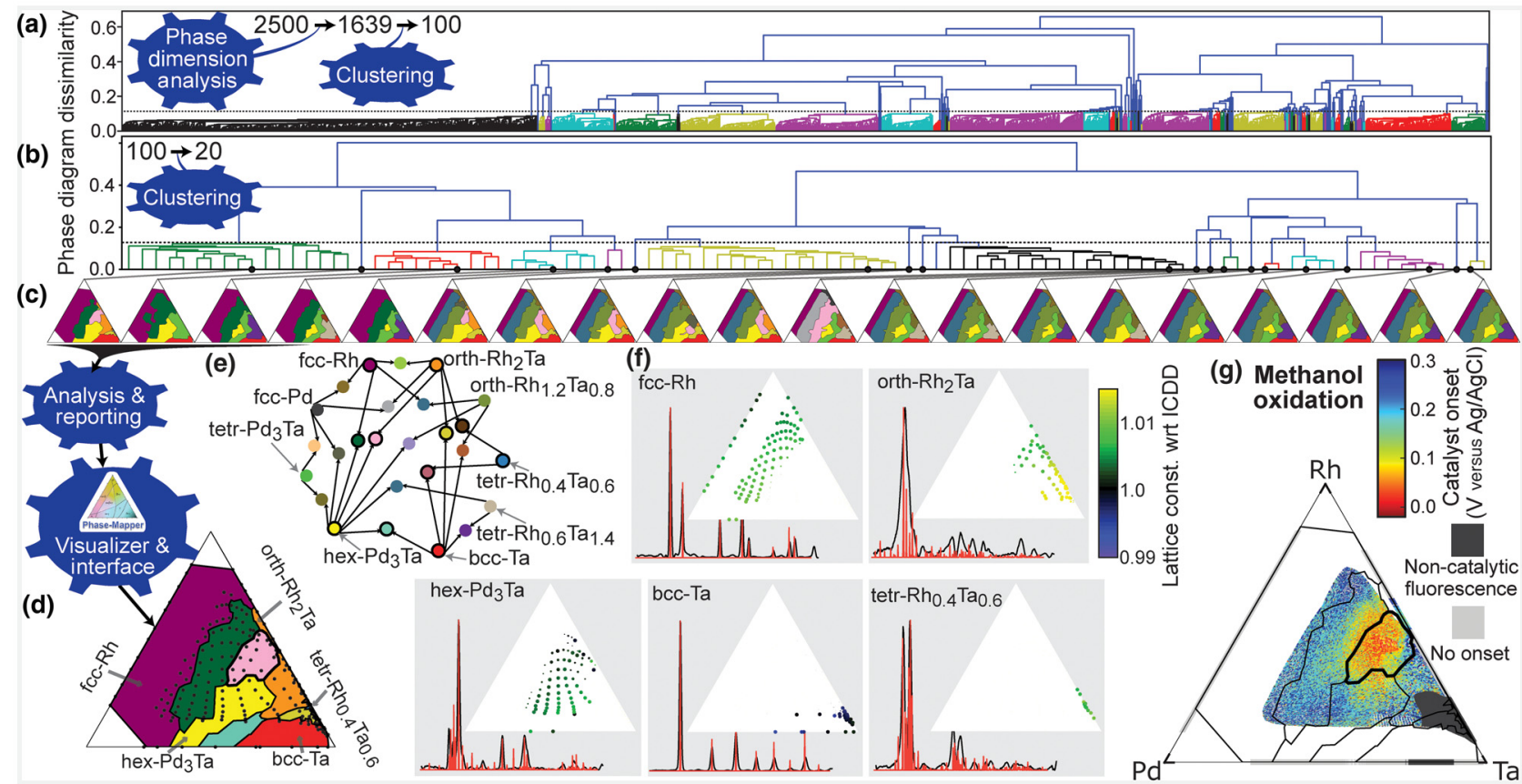

Figure 4. CRYSTAL's solution to the Pd-Rh-Ta catalyst system. (a) CRYSTAL automatically generates 2500 phase diagrams in parallel from which the Phase Dimension Analysis bot identifies 1639 valid solutions and the Clustering bot identifies 100 representative solutions for additional refinement. (b) From the 100 refined phase diagrams, CRYSTAL automatically identifies the span of solutions with different physical meaning, which is 20 phase diagrams in this case. (c) The selected 20 phase diagrams that represent their respective clusters. (d) The final solution resulting from expert consideration of CRYSTAL's report. The expert user also provided minor manual refinement of the phase diagram, in particular small phase field boundary adjustments in composition regions with sparse measurement data. (e) Color scheme for the phase fields where the single-phase fields are labeled and phase combinations are denoted by linkages. The 11 phase fields marked with a black circle appear in the final solution. (f) The basis patterns for the final solution with stick patterns from the International Center for Diffraction Data shown in red. Composition maps of the relative lattice constant for each phase reveal alloying-based shifts due to the different atomic radii of the elements ( $\mathrm{Ta}>\mathrm{Pd}>\mathrm{Rh}$ ). The dot size denotes the phase concentration. $(\mathrm{g})$ The methanol oxidation onset potential for the ternary and binary composition spaces where $\mathrm{Rh}-\mathrm{Ta}$ is the only binary to exhibit catalytic activity. The overlay of the final solution's phase field boundaries reveals that the best activity (lowest onset potential) is observed in the mixed orth- $R h_{2} T a+$ hex- $-\mathrm{Pd}_{3} T a$ phase field.

Triangulation. The Connectivity constraint states that the sample locations $n$ for which $\sum_{m} \mathbf{H}_{n, k}^{m}$ is larger than zero must form a connected component in graph $G$, and that phase fields consisting of a unique combination of phases must similarly form a connected component. The Phase and Phase Field Connectivity bot rectifies the connectivity constraints in a lazy and iterative manner. See also Supplementary Information.

\section{Phase Matching and Phase Dimensionality Analysis bots}

The Phase Matching bot matches the basis patterns produced by the IAFD module, as part of each solution, to known crystal structure patterns from the ICDD. Fitting an ICDD-derived pattern to each basis pattern (see Supplementary Information for details) provides the additional opportunity to threshold the loss such that if no ICDD-derived pattern sufficiently matches the basis pattern, then the basis pattern may be describing a new phase. The matching of a single ICDD entry to multiple basis patterns in a single solution is an indication that the $K$ (number of phases) of the solution is too large and thus the solution is invalid. Based on this concept, the CRYSTAL planner monitors Phase Dimensionality Analysis results to determine the maximum number of phases to consider for the given system. The CRYSTAL planner runs IAFD configurations with an increasing number of phases until the resulting phase diagrams have an ICDD entry assigned to more than one basis pattern or the valid solution rate becomes vanishingly small, providing automatic determination of the upper bound on the number of phases (basis patterns).

\section{Phase Diagram Clustering and Analysis \& Reporting bots}

The IAFD bot produces physically meaningful phase diagrams, whose phases are labeled using the ICDD, for the known phases. CRYSTAL runs the IAFD module in parallel (in this paper we report 500 runs for a given number of phases but in general 100 or fewer runs is sufficient) to produce candidate phase diagrams that require automated phase diagram analysis and consolidation. The Clustering bot takes as input the set of solutions produced by parallel runs of the IAFD module and outputs a small set of representative candidate solutions. This bot uses hierarchical agglomerative clustering based on 
pair-wise phase diagram dissimilarity with automated thresholding. A distance metric between phase diagrams is defined as the across-sample average of the dissimilarity of the pair of phase fields in which the sample resides in the pair of phase diagrams. The phase field for a given sample is defined as the set of basis patterns activated for that sample, which is labeled according to the ICDD phase matching results, and the comparison of the resulting pair of ICDD phase sets is performed under consideration that multiple ICDD patterns may match a given basis pattern within a predefined tolerance. Hierarchical clustering of phase diagram solutions provides (i) 100 representative solutions from the initial runs of IAFD and (ii) a portfolio of unique phase diagrams from the 100 refined solutions where the number of clusters is determined through automated thresholding based on unique sets of ICDD patterns. The set of representative candidate solutions identified by the clustering bot are provided to either the CRYSTAL Planner for solution refinement by the IAFD module, as initial solutions, or to the Analysis \& Reporting bot to generate phase diagrams and other visualizations for human-expert inspection.

\section{Pd-Rh-Ta experiments}

The Pd-Rh-Ta system was chosen for investigation based on the use of Pd in catalysts for alcohol oxidation in alkaline electrolytes $^{[22]}$ and recent success improving the methanol oxidation reactivity of $\mathrm{Pt}$ by combining it with $\mathrm{Ta}$, where surface sub-oxides of Ta appeared to lower the adsorption of $\mathrm{CO}$ and thus mitigate catalyst poisoning. ${ }^{[23]}$ The $197 \mathrm{XRD}$ and XRF measurements were acquired on four co-sputtered thin film composition libraries: the Pd-Rh-Ta ternary library and one for each binary system (the edges of the composition triangle). The XRF measurements provide the mapping from the physical location on the substrate to the Pd-Rh-Ta ternary composition space, with measurement details and data processing as described in Ref. 24.

The catalytic activity of each of the four composition spread thin films was mapped using the high-throughput fluorescencebased screening, and the results for the Pd-Rh-Ta film are shown in Fig. 1(b) with false-color images of fluorescence intensity showing background-subtracted images from a charge-coupled device camera, as previously described. ${ }^{[23,25]}$ The $\mathrm{N}_{2}$-sparged aqueous electrolyte contained $3 \mathrm{mM}$ quinine (fluorescent indicator) and $0.1 \mathrm{M}$ potassium triflate (supporting electrolyte). An initial voltage sweep in this electrolyte without methanol was used to identify any regions exhibiting fluorescence, which could be due to film oxidation or oxidative corrosion, prompting our labeling of these composition regions as "unstable." A solution with the addition of $5 \mathrm{M}$ methanol was then used for screening catalysis of the methanol oxidation reaction. As standard practice, the experiment was repeated three times with fresh electrolyte and similar results were obtained, Figs. 1(b) and 4(g) showing the results from the first of these voltage sweeps. In all of these experiments, the voltage sweep was performed from 0.4 to $+0.5 \mathrm{~V}$ versus $\mathrm{Ag} /$
$\mathrm{AgCl}$ at a scan rate of $0.05 \mathrm{~V} / \mathrm{s}$. By setting a fluorescence intensity threshold, the onset potential associated with each pixel in the library image was determined and the XRF-based composition map was used to map the onset potential data to composition space, as shown in Fig. 4(g).

\section{Phase mapping for catalyst discovery in the Pd-Rh-Ta system}

The Pd-Rh-Ta system poses substantial phase mapping challenges due to strongly overlapped features in its phases' XRD patterns as well as substantial alloying-based peak shifting, which are compounded by experimental noise in the thin-film XRD measurements. CRYSTAL generated a total of 2500 phase maps (500 phase maps per configuration with a number of phases $K=3,4,5,6$, and 7). As shown in Table I, $100 \%$ of the composition points, phases, and phase fields meet the physical constraints imposed by the algorithmic bots. For comparison, an analogous 2500 runs were performed using AgileFD and NMF, with Table I revealing some constraint satisfaction, but not sufficient to produce any physically meaningful solutions. Comparison was also made with $\mathrm{NMF}_{K}$, a recently reported algorithm that involves clustering of NMF components to identify basis patterns, ${ }^{[26]}$ which produced a single $K=5$ solution for which the constraint satisfaction rates exceed that of NMF and AgileFD but still do not provide a physically meaningful phase diagram where all constraints are satisfied. Comparison of this $\mathrm{NMF}_{K}$ solution with that of Fig. 4 is shown in Fig. S1, revealing a substantially different interpretation of the data.

CRYSTAL continued processing of its initial 2500 phase diagrams via the Phase Dimension Analysis bot, which determined that the system contains no more than $K=6$ phases and passed 1639 valid solutions to the Clustering bot, which identified 100 representative solutions using hierarchical agglomerative clustering based on pairwise phase diagram dissimilarity. After further refining the 100 solutions using the IAFD bots, the hierarchical Clustering bot, using automated thresholding, identified 20 representative phase diagrams that represent the span of different data interpretations. These phase diagrams were passed to the Analysis \& Reporting bot to produce phase diagram visualizations and composition maps of the phase fields and lattice constant shifts, which are readily interpretable by an expert [Figs. 1(d)-1(f) and 4]. The expert user analyzed and compared the 20 candidate phase diagrams, eliminating 15 candidate phase diagrams since they do not meet subtle criteria based on prior knowledge specific to the $\mathrm{Pd}-\mathrm{Rh}-\mathrm{Ta}$ system. In this case, this prior knowledge was from a previous analysis of the Pd-Rh binary line where a single face centered cubic (fcc) seven-phase diagram was analyzed. While this prior knowledge was used to screen candidate solutions in the present work, this type of knowledge can be used to initialize and/or constrain IAFD, as described previously for AgileFD. ${ }^{[19,20]}$ Other types of prior knowledge may also be incorporated, which may require the development of new algorithmic bots, another motivation for building CRYSTAL as a 
Table I. Comparison of constraint satisfaction for solutions generated by different algorithms.

\begin{tabular}{|c|c|c|c|c|c|c|}
\hline K (\# basis patterns) & & 3 & 4 & 5 & 6 & 7 \\
\hline \multirow[t]{4}{*}{ CRYSTAL } & Gibbs & $100 \%$ & $100 \%$ & $100 \%$ & $100 \%$ & $100 \%$ \\
\hline & Gibbs-Alloy & $100 \%$ & $100 \%$ & $100 \%$ & $100 \%$ & $100 \%$ \\
\hline & Pure phase connectivity & $100 \%$ & $100 \%$ & $100 \%$ & $100 \%$ & $100 \%$ \\
\hline & Phase field connectivity & $100 \%$ & $100 \%$ & $100 \%$ & $100 \%$ & $100 \%$ \\
\hline \multirow[t]{4}{*}{ AgileFD } & Gibbs & $100 \%$ & $74.93 \%$ & $60.20 \%$ & $47.46 \%$ & $37.46 \%$ \\
\hline & Gibbs-Alloy & $52.66 \%$ & $32.36 \%$ & $19.05 \%$ & $11.71 \%$ & $7.88 \%$ \\
\hline & Pure phase connectivity & $63.67 \%$ & $49.95 \%$ & $16.68 \%$ & $7.03 \%$ & $2.31 \%$ \\
\hline & Phase field connectivity & $33.99 \%$ & $32.29 \%$ & $28.58 \%$ & $34.55 \%$ & $49.38 \%$ \\
\hline \multirow[t]{4}{*}{ NMF } & Gibbs & $100 \%$ & $79.01 \%$ & $64.35 \%$ & $55.01 \%$ & $47.24 \%$ \\
\hline & Gibbs-Alloy & $100 \%$ & $79.01 \%$ & $64.35 \%$ & $55.01 \%$ & $47.24 \%$ \\
\hline & Pure phase connectivity & $51.60 \%$ & $29.70 \%$ & $23.68 \%$ & $11.17 \%$ & $1.94 \%$ \\
\hline & Phase field connectivity & $28.73 \%$ & $26.37 \%$ & $24.73 \%$ & $39.22 \%$ & $56.42 \%$ \\
\hline \multirow[t]{4}{*}{$\mathrm{NMF}_{\mathrm{K}}$} & Gibbs & NA & NA & $87 \%$ & NA & NA \\
\hline & Gibbs-Alloy & NA & NA & $77 \%$ & NA & NA \\
\hline & Pure phase connectivity & NA & NA & $40 \%$ & NA & NA \\
\hline & Phase field connectivity & NA & NA & $50 \%$ & NA & NA \\
\hline
\end{tabular}

For each number of basis patterns $(K), 500$ random initializations were used to generate a set of solutions, which were then evaluated for compliance with four physical constraints. The percentage of samples (Gibbs and Gibbs-Alloy), percentage of phases (Pure phase connectivity), and percentage of phase fields (Phase field connectivity) are shown assuming a threshold value of $10^{-6}$ on phase activations (values of $\mathbf{H}$ ). While AgileFD and NMF solutions satisfy constraints for

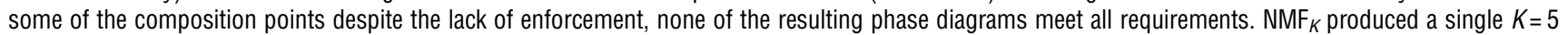
solution, which satisfies constraints better than NMF and AgileFD but similarly does not provide physically meaningful phase diagrams where all constraints are satisfied. Due to its constraint enforcement, CRYSTAL produces solutions which meet all of the physical requirements.

network of bots that can be adapted for specific research tasks and expanded to incorporate new modes of reasoning. After applying this filter, the expert user selected the final phase diagram remaining five phase diagrams, which we note could have also been automatically selected via a voting method since the selected phase diagram represents the hierarchical cluster containing 17 refined solutions, more than any other remaining cluster [Fig. 4(c)].

In addition to identifying complete solubility of $\mathrm{Pd}$ and $\mathrm{Rh}$ into the fcc structure, this phase diagram also indicates substantial alloying in the intermetallic phases [Figs. 4(d)-4(f)]. For each phase, the lattice constant variation with composition matches expectations based on the metallic radii of the elements. For the three non-cubic phases, the observed $<1 \%$ lattice expansions are well modeled by the isometric peak shifting model. The hex- $\mathrm{Pd}_{3} \mathrm{Ta}$ phase exhibits the largest alloying extent of these phases, with up to 30 at.\% of the smaller $\mathrm{Rh}$ and up to 35 at.\% excess of the larger Ta leading to lattice expansions $<0.7 \%$.

CRYSTAL's phase diagram enabled insightful interpretation of the catalytic activity in the Pd-Rh-Ta system. Figure 4(g) shows the result of a high-throughput screening of the Pd-Rh-Ta libraries for methanol oxidation, a critical reaction for direct methanol fuel cells traditionally addressed with Pt-based catalysts. ${ }^{[27]}$ While many of the compositions exhibit inactivity or instability under the reaction conditions, selected Pd-Rh-Ta compositions exhibit an activity that is on par with the best Pt-based catalysts evaluated by this high-throughput method. On the Rh-Ta binary line, the orth- $\mathrm{Rh}_{2} \mathrm{Ta}$ phase provides the highest catalytic activity. The methanol oxidation onset potential is further lowered by $0.2 \mathrm{~V}$ to approximately $0.5 \mathrm{~V}$ versus $\mathrm{RHE}$ via mixing orth- $\mathrm{Rh}_{2} \mathrm{Ta}$ with an alloy of hex- $\mathrm{Pd}_{3} \mathrm{Ta}$ at a composition $\mathrm{Pd}_{0.17} \mathrm{Rh}_{0.33} \mathrm{Ta}_{0.5}$. Such combinations of catalyst materials have recently been proposed for overcoming historical barriers that limit catalyst performance for multi-step reactions, ${ }^{[28,29]}$ and indeed the activity of this multiintermetallic catalyst is quite remarkable. The best thin film catalysts for methanol oxidation that have been identified by this technique include the Pt-Ta intermetallics with an onset potential of $120 \mathrm{mV}$ versus $\mathrm{Ag} / \mathrm{AgCl},{ }^{[23]}$ and a family of Pt-based fcc alloys including binary alloys with $\mathrm{Ru}, \mathrm{In}, \mathrm{Sn}$, and $\mathrm{Zn}$, which have onset potentials between 0 and $40 \mathrm{mV}$ versus $\mathrm{Ag} /$ 
AgCl. ${ }^{[30]}$ The $\mathrm{Pd}_{0.17} \mathrm{Rh}_{0.33} \mathrm{Ta}_{0.5}$ catalyst is the only known Pt-free catalyst with onset potential in this range. The onset potential of $0.5 \mathrm{~V}$ versus RHE is also within the range of onset overpotentials observed with Pd-based catalyst in alkaline electrolytes, ${ }^{[22]}$ and given the inactivity of $\mathrm{Pd}$ and $\mathrm{Rh}$ in the weak acidic electrolyte used in our experiments, the Ta-based intermetallics appear to enable the activity at lower $\mathrm{pH}$, opening a new direction for catalyst development and a pathway for the further development of Pt-free catalysts.

The discovery of this multiphase catalyst highlights both the power of high-throughput materials science and the effectiveness of AI techniques for integrating multiple knowledge sources to provide meaningful solutions. By teaching CRYSTAL to reason about phase diagrams, we have for the first time automated the generation and exploration of alternative data models, demonstrating the ability of AI systems to accelerate phase mapping and providing a novel datainterpretation approach for materials sciences and beyond.

\section{Supplementary material}

The supplementary material for this article can be found at https://doi.org/10.1557/mrc.2019.50

\section{Acknowledgments}

This work was supported by NSF awards CCF-1522054 and CNS-0832782 (Expeditions), CNS-1059284 (Infrastructure), and IIS-1344201 (INSPIRE); ARO awards W911NF-14-1-0498 and W911NF-17-1-0187; AFOSR Multidisciplinary University Research Initiatives (MURI) Program FA9550-18-1-0136, Toyota Research Institute award; and US DOE Award No. DE-SC0004993. Use of SSRL is supported by DOE Contract No. DE-AC02-76SF00515. Use of CHESS is supported by the NSF award DMR-1332208. The authors thank A. Mehta, D. G. Van Campen, M. Tague, and D. Dale for assistance with data collection.

\section{Author contributions}

The authors' contributions are as follows: C.P.G., R.B.vD., and J.M.G. conceived and managed the project. C.P.G. conceived CRYSTAL'S multiple knowledge source approach. J.Ba., JBj., C.P.G., and Y.X. designed the bots' algorithms. J.Ba., C.P.G., J.M.G., B.H.R., and Y.X. designed the Diagram Rendering bot. J.Ba. implemented the IAFD bots, phase matching bot, and phase analysis bot. B.H.R. implemented the diagram rendering algorithm, and Analysis \& Reporting and Visualizer \& Interface bots. S.K. performed the comparison with $\mathrm{NMF}_{K}$. R.A.B. assisted with programming in several components of CRYSTAL. R.B.vD. and J.M.G. acquired Pd-Rh-Ta data, and S.K.S. and J.M.G. acquired $\mathrm{Nb}-\mathrm{Cu}-\mathrm{V}$ data with assistance as noted in the Acknowledgments. S.K.S. and J.M.G. served as human experts for both systems. C.P.G. and J.M.G. were the primary authors of the manuscript. S.A., J.Ba., J.Bj., C.P.G., J.G.M., B.H.R., and Y.X. were the primary authors of the Methods and Supplementary Information.

\section{Data availability}

The raw data for the Pd-Rh-Ta along with CRYSTAL's results and reports will be available at http://www.udiscover.it/resources/data/. Further documentation and source code for IAFD can be found at http://www.udiscover.it/resources/software/.

\section{Author information}

The authors declare no competing financial interests. Correspondence should be addressed to gomes@cs.cornell. edu and gregoire@caltech.edu.

\section{References}

1. Artificial intelligence. Science 349, 248 (2015).

2. D. Silver, J. Schrittwieser, K. Simonyan, I. Antonoglou, A. Huang, A. Guez, T. Hubert, L. Baker, M. Lai, A. Bolton, Y. Chen, T. Lillicrap, F. Hui, L. Sifre, G. van den Driessche, T. Graepel, and D. Hassabis: Mastering the game of Go without human knowledge. Nature 550, 354 (2017).

3. D.P. Tabor, L.M. Roch, S.K. Saikin, C. Kreisbeck, D. Sheberla, J.H. Montoya, S. Dwaraknath, M. Aykol, C. Ortiz, H. Tribukait, C. Amador-Bedolla, C.J. Brabec, B. Maruyama, K.A. Persson, and A. Aspuru-Guzik: Accelerating the discovery of materials for clean energy in the era of smart automation. Nat. Rev. Mater. 3, 5 (2018).

4. P. De Luna, J. Wei, Y. Bengio, A. Aspuru-Guzik, and E. Sargent: Use machine learning to find energy materials. Nature 552, 23 (2017).

5. R. Ramprasad, R. Batra, G. Pilania, A. Mannodi-Kanakkithodi, and C. Kim: Machine learning in materials informatics: recent applications and prospects. Nat. Comput. Mater. 3, 54 (2017).

6. P. Nikolaev, D. Hooper, F. Webber, R. Rao, K. Decker, M. Krein, J. Poleski, R. Barto, and B. Maruyama: Autonomy in materials research: a case study in carbon nanotube growth. Nat. Comput. Mater. 2, 16031 (2016).

7. E. Smalley: Al-powered drug discovery captures pharma interest. Nat. Biotechnol. 35, 604 (2017).

8. R.D. King, K.E. Whelan, F.M. Jones, P.G.K. Reiser, C.H. Bryant, S.H. Muggleton, D.B. Kell, and S.G. Oliver: Functional genomic hypothesis generation and experimentation by a robot scientist. Nature 427, 247 (2004).

9. M.L. Green, C.L. Choi, J.R. Hattrick-Simpers, A.M. Joshi, I. Takeuchi, S.C. Barron, E. Campo, T. Chiang, S. Empedocles, J.M. Gregoire, A.G. Kusne J. Martin, A. Mehta, K. Persson, Z. Trautt, J.V. Duren, and A. Zakutayev: Fulfilling the promise of the materials genome initiative with highthroughput experimental methodologies. Appl. Phys. Rev. 4, 011105 (2017).

10. A.G. Kusne, T. Gao, A. Mehta, L. Ke, M.C. Nguyen, K.-M. Ho, V. Antropov, C.-Z. Wang, M.J. Kramer, C. Long, and I. Takeuchi: On-the-fly machinelearning for high-throughput experiments: search for rare-earth-free permanent magnets. Sci. Rep. 4, 6367 (2014).

11. E. Reddington, A. Sapienza, B. Gurau, R. Viswanathan, S. Sarangapani, E.S. Smotkin, and T.E. Mallouk: Combinatorial electrochemistry: a highly parallel, optical screening method for discovery of better electrocatalysts. Science 280, 1735 (1998).

12. J.R. Hattrick-Simpers, J.M. Gregoire, and A.G. Kusne: Perspective: composition-structure-property mapping in high-throughput experiments: turning data into knowledge. APL Mater. 4, 053211 (2016).

13. L.A. Baumes, M. Moliner, N. Nicoloyannis, and A. Corma: A reliable methodology for high throughput identification of a mixture of crystallographic phases from powder x-ray diffraction data. Cryst. Eng. Comm. 10, 1321 (2008).

14.D.D. Lee and H.S. Seung: Learning the parts of objects by non-negative matrix factorization. Nature 401, 788 (1999).

15. C.J. Long, D. Bunker, X. Li, V.L. Karen, and I. Takeuchi: Rapid identification of structural phases in combinatorial thin-film libraries using $x$-ray diffraction and non-negative matrix factorization. Rev. Sci. Instrum. 80, 103902 (2009). 
16. A.G. Kusne, D. Keller, A. Anderson, A. Zaban, and I. Takeuchi: Highthroughput determination of structural phase diagram and constituent phases using GRENDEL. Nanotechnology 26, 444002 (2015).

17. R. LeBras, T. Damoulas, J.M. Gregoire, A. Sabharwal, C.P. Gomes, and R.B. van Dover: Constraint Reasoning and Kernel Clustering for Pattern Decomposition with Scaling, in Principles and Practice of Constraint Programming - CP 2011: 17th International Conference, CP 2011, Perugia, Italy, September 12-16, 2011. Proceedings, edited by J. Lee (Springer Berlin Heidelberg, Berlin, Heidelberg, 2011), p. 508.

18. A. Cichocki, R. Zdunek, A.H. Phan, and S. Amari: Nonnegative Matrix and Tensor Factorizations: Applications to Exploratory Multi-way Data Analysis and Blind Source Separation (John Wiley \& Sons, Chichester, West Sussex, UK, 2009).

19.P. Smaragdis: Non-negative Matrix Factor Deconvolution; Extraction of Multiple Sound Sources from Monophonic Inputs, in Independent Component Analysis and Blind Signal Separation: Fifth International Conference, ICA 2004, Granada, Spain, September 22-24, 2004. Proceedings, edited by C. G. Puntonet and A. Prieto (Springer Berlin Heidelberg, Berlin, Heidelberg, 2004), p. 494.

20.S.K. Suram, Y. Xue, J. Bai, R. Le Bras, B. Rappazzo, R. Bernstein, J. Bjorck, L. Zhou, R.B. van Dover, C.P. Gomes, and J.M. Gregoire: Automated phase mapping with AgileFD and its application to light absorber discovery in the V-Mn-Nb oxide system. ACS Comb. Sci. 19, 37 (2017).

21.J. Bai, J. Bjorck, Y. Xue, S.K. Suram, J. Gregoire, and C. Gomes: Relaxation methods for constrained matrix factorization problems: solving the phase mapping problem in materials discovery, in International Conference on $\mathrm{Al}$ and $\mathrm{OR}$ Techniques in Constraint Programming for Combinatorial Optimization Problems (Springer 2017), p. 104.

22. C. Bianchini and P.K. Shen: Palladium-based electrocatalysts for alcohol oxidation in half cells and in direct alcohol fuel cells. Chem. Rev. 109, 4183 (2009).

23. J.M. Gregoire, M.E. Tague, S. Cahen, S. Khan, H.C.D. Abruña, F.J. DiSalvo, and R.B. van Dover: Improved fuel cell oxidation catalysis in $\mathrm{Pt}_{1-\mathrm{x}} \mathrm{Ta}_{\mathrm{x}}$. Chem. Mater. 22, 1080 (2009).

24. J.M. Gregoire, D. Dale, A. Kazimirov, F.J. DiSalvo, and R.B. van Dover: High energy $x$-ray diffraction/x-ray fluorescence spectroscopy for highthroughput analysis of composition spread thin films. Rev. Sci. Instrum. 80, 123905 (2009).

25. J. Jin, M. Prochaska, D. Rochefort, D. Kim, L. Zhuang, F. Disalvo, R. Vandover, and H. Abruna: A high-throughput search for direct methanol fuel cell anode electrocatalysts of type PtxBiyPbz. Appl. Surf. Sci. 254, 653 (2007).

26. V. Stanev, V.V. Vesselinov, A.G. Kusne, G. Antoszewski, I. Takeuchi, and B.S. Alexandrov: Unsupervised phase mapping of $x$-ray diffraction data by nonnegative matrix factorization integrated with custom clustering. $n p j$ Comput. Mater. 4, 43 (2018).

27. H. Liu, C. Song, L. Zhang, J. Zhang, H. Wang, and D.P. Wilkinson: A review of anode catalysis in the direct methanol fuel cell. J. Power Sources 155, 95 (2006).

28. M. Andersen, A.J. Medford, J.K. Nørskov, and K. Reuter: Scaling-relationbased analysis of bifunctional catalysis: the case for homogeneous bimetallic alloys. ACS Catal. 7, 3960 (2017).

29. E. Casado-Rivera, Z. Gál, A.C.D. Angelo, C. Lind, F.J. DiSalvo, and H.D. Abruña: Electrocatalytic oxidation of formic acid at an ordered intermetallic PtBi surface. ChemPhysChem 4, 193 (2003).

30. M.E. Tague, J.M. Gregoire, A. Legard, E. Smith, D. Dale, R. Hennig, F.J. DiSalvo, R.B. van Dover, and H.D. Abruña: High throughput thin film Pt-M alloys for fuel electrooxidation: low concentrations of $\mathrm{M}(\mathrm{M}=\mathrm{Sn}, \mathrm{Ta}, \mathrm{W}$, $\mathrm{Mo}, \mathrm{Ru}, \mathrm{Fe}, \mathrm{In}, \mathrm{Pd}, \mathrm{Hf}, \mathrm{Zn}, \mathrm{Zr}, \mathrm{Nb}, \mathrm{Sc}, \mathrm{Ni}, \mathrm{Ti}, \mathrm{V}, \mathrm{Cr}, \mathrm{Rh})$. J. Electrochem. Soc. 159, F880 (2012). 